Research Article

\title{
DFT Calculations of Hydrogen Adsorption inside Single-Walled Carbon Nanotubes
}

\author{
Igor K. Petrushenko iD \\ Irkutsk National Research Technical University, 83 Lermontov St., Irkutsk 664074, Russia \\ Correspondence should be addressed to Igor K. Petrushenko; igor.petrushenko@istu.edu
}

Received 28 August 2017; Accepted 7 November 2017; Published 18 January 2018

Academic Editor: Yuanshi Li

Copyright (C) 2018 Igor K. Petrushenko. This is an open access article distributed under the Creative Commons Attribution License, which permits unrestricted use, distribution, and reproduction in any medium, provided the original work is properly cited.

DFT calculations have been performed to study noncovalent interactions of a hydrogen molecule and single-walled carbon nanotubes (SWCNTs) of various diameters. Understanding these interactions is crucial for the development of systems for hydrogen storage and delivery. The barrier and barrier-free introduction of a hydrogen molecule into SWCNTs is observed. It has been found that hydrogen molecules bind differently onto SWCNTs, depending on their diameters and the orientation of an $\mathrm{H}_{2}$ molecule inside the SWCNT. The binding inside SWCNTs with small diameters $((3,3) ;(4,4))$ is very unfavorable; the opposite situation is in the case of larger $((5,5) ;(6,6))$ SWCNTs. Finally, in the case of $((7,7) ;(8,8))$ SWCNTs, the hydrogen binding energies decrease, and their values approach to those of graphene.

\section{Introduction}

In recent years, numerous studies on the potential applications of single-walled carbon nanotubes (SWCNTs) [1] in various fields including the development of novel organic electronic materials, chemical sensors, and vehicles for organ-specific drug delivery and other therapeutic processes have been carried out [2-10]. Noncovalent interactions of small molecules and SWCNTs are pivotal to many of these applications [11-13].

The ultimate exploiting the noncovalent functionalization of SWCNTs will require detailed information on the factors that affect these interactions. Therefore, many experimentalists and theorists have examined noncovalent interactions of small molecules with the exterior walls of SWCNTs and the encapsulation of molecules inside SWCNTs [14-16]. Among these molecules, one of the most important is hydrogen. Hydrogen is a perfect alternative energy source. It is abundant in nature, has low environmental effects, and finally, its combustion produces only water and heat. Recently, the scientific efforts have been focused on finding the efficient, safe, and cheap method to store hydrogen for the possible usage in large-scale applications [17-20]. There are different technologies for hydrogen storage such as liquefaction, metal hybrids, and physisorption. An effective method of keeping is relied on both the ability to simply place hydrogen in the "tank" and the facility to release it for potential use. From this point of view, it is worth studying SWCNTs, hollow nanocontainers, to ascertain whether it is possible to use them efficiently for hydrogen storage. Although some papers study hydrogen adsorption onto outer walls of SWCNTs [21], lead(II)- and aluminium-based metal-organic framework [22, 23], and boron nitride nanotubes [24], there is only one paper, to the best of our knowledge, which studies hydrogen confined into carbon nanotubes with very small diameters [25]. Thus, there exists a demand to study interactions of hydrogen molecules and interior surfaces of SWCNTs of various diameters.

Inspired by the above considerations, in this paper, we quantify the noncovalent interactions between hydrogen molecules and the inner walls of SWCNTs as well as graphene by techniques based on the dispersion-corrected density functional theory (DFT-D3), which has been successfully applied to predict adsorption properties of many systems with high reliability and low computational efforts [26-28]. 


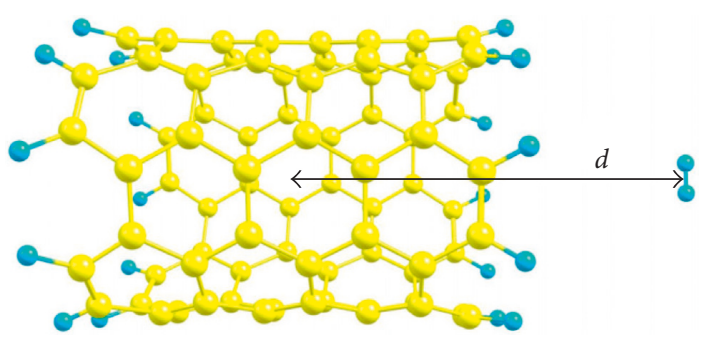

(a)

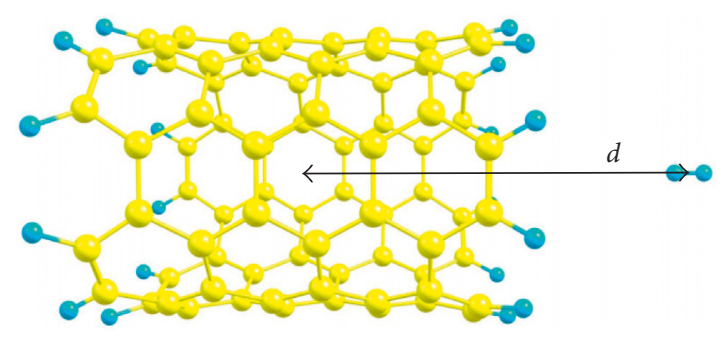

(b)

Figure 1: Perpendicular (a) and parallel (b) orientations of an $\mathrm{H}_{2}$ molecule in relation to the axis of $(5,5)$ SWCNT as an example. Small balls (blue) are hydrogen, and large balls (yellow) are carbon.

\section{Computational Methods}

All calculations were carried out using Orca 3.0.3 program package [29]. The dispersion-corrected (as proposed by Grimme) GGA PBE-D3 functional [30-32] together with the SVP basis set [33] was employed. This functional has been demonstrated to be reliable and commonly used in the studies of adsorption phenomena $[34,35]$. We consider various armchair nanotubes as models of $(n, n)$ SWCNTs, $n=3-8$. The following models of SWCNTs are used: $\mathrm{C}_{48} \mathrm{H}_{12}$ $(3,3), \mathrm{C}_{64} \mathrm{H}_{16}(4,4), \mathrm{C}_{80} \mathrm{H}_{20}(5,5), \mathrm{C}_{96} \mathrm{H}_{24}(6,6), \mathrm{C}_{112} \mathrm{H}_{28}(7,7)$, and $\mathrm{C}_{138} \mathrm{H}_{32}(8,8)$. It was earlier predicted that the $(2,2)$ armchair SWCNT is the carbon nanotube with the smallest possible diameter of $3 \AA$ [36]. However, that type of nanotubes was formed inside a MWCNT of a larger diameter. Therefore, in this study, we use the $(3,3)$ model as the smallest one as predicted in [37]. The coronene molecule $\left(\mathrm{C}_{24} \mathrm{H}_{12}\right)$ is adopted as a graphene model system (Figure 1$)$. It was stated that coronene is the smallest molecule that resembles the properties of graphene [38].

First, pristine graphene, all SWCNTs models, and a hydrogen molecule were fully optimized at the PBE-D3/SVP level of theory. The geometries of these structures were then frozen. Second, we fixed the perpendicular orientation of a hydrogen molecule relative to the axis of the employed SWCNTs and simulated the introduction of the molecule inside the SWCNTs. Third, we fixed the parallel orientation of hydrogen relative to the studied nanotubes and repeated the latter step. The distance $(d)$ between $\mathrm{H}_{2}$ and the center of studied SWCNTs is defined as the length of a normal line dropped from the hydrogen center of mass to the center of SWCNTs. Finally, we varied $d$ from 10 to $0 \AA$ and calculated single-point interaction energies $\left(E_{i, m}, m=0-10\right)$ using the following equation:

$$
E_{i, m}=E_{m}\left(\frac{\mathrm{H}_{2}}{\mathrm{SWCNT}}\right)-E\left(\mathrm{H}_{2}\right)-E(\mathrm{SWCNT}),
$$

where $E_{m}\left(\mathrm{H}_{2} / \mathrm{SWCNT}\right), E_{\mathrm{sp}}\left(\mathrm{H}_{2}\right)$, and $E_{\mathrm{sp}}(\mathrm{SWCNT})$ denote single-point energy of the hydrogen/SWCNT complex and the equilibrium energies of the hydrogen molecule and SWCNT, respectively.

As $E_{i}$ will be used in the rest of the paper for the description of interactions between $\mathrm{H}_{2}$ and SWCNTs at the SWCNT center, we adopt $E_{i, 0}$ value. The interactions of $\mathrm{H}_{2}$ molecules with SWCNTs and graphene are also characterized by binding energies $\left(E_{b}\right)$. It accounts for the relaxation of the hydrogen molecule and SWCNT (graphene) and can be calculated as

$$
E_{b}=E\left(\frac{\mathrm{H}_{2}}{\mathrm{SWCNT}}\right)-E\left(\mathrm{H}_{2}\right)-E(\mathrm{SWCNT}),
$$

where $E\left(\mathrm{H}_{2} / \mathrm{SWCNT}\right), E\left(\mathrm{H}_{2}\right)$, and $E(\mathrm{SWCNT})$ denote the equilibrium energies of the hydrogen/SWCNT complex, the hydrogen molecule, and SWCNT, respectively. The former is defined as the single-point interaction energy between optimized hydrogen and SWCNTs. This is in contrast to the binding energies, which account for the relaxation of the involved complexes.

\section{Results and Discussions}

3.1. Introduction of a Hydrogen Molecule inside a Series of SWCNTs. In order to understand the fundamental interactions between hydrogen molecules and SWCNTs, we introduce a single $\mathrm{H}_{2}$ molecule inside a series of nanotubes with different diameters (from 4.140 to $10.907 \AA$ ) to have the opportunity to point out all phenomena that appear inside SWCNTs of small and large diameters. For this aim, we theoretically study a step-by-step insertion of a hydrogen molecule along the nanotube axis in two orientations relative to the axis (parallel and perpendicular ones), using PBE-D3 (Figure 1). Here, we (1) quantify the energetics for the entry of hydrogen into SWCNTs under study to figure out the possibility of SWCNTs loading as well as the feasibility of formation of endohedral complexes, (2) evaluate the noncovalent interactions between hydrogen molecules and inner walls of SWCNTs as well as graphene, and (3) show the differences in $\mathrm{H}_{2}$ behavior upon interactions with SWCNTs of various diameters.

The interaction energies $\left(E_{i, m}\right)$ as a function of distance (d) from the center of studied SWCNTs to the center of mass of a hydrogen molecule are plotted for the two aforementioned orientations (Figure 1). The $d$ value of zero corresponds to the center of a SWCNT. For the $(3,3)$ and $(4,4)$ SWCNTs, we can see that there exists an entry barrier for an $\mathrm{H}_{2}$ encapsulation in either orientation. The $d$ of $7 \AA$ for the $(3,3)$ SWCNT and $6.5 \AA$ for $(4,4)$ SWCNT corresponds to the local minima caused by the van der Waals interactions between hydrogen and SWCNTs. As is expected, the energy barrier for the hydrogen introduction is larger for the $(3,3)$ 


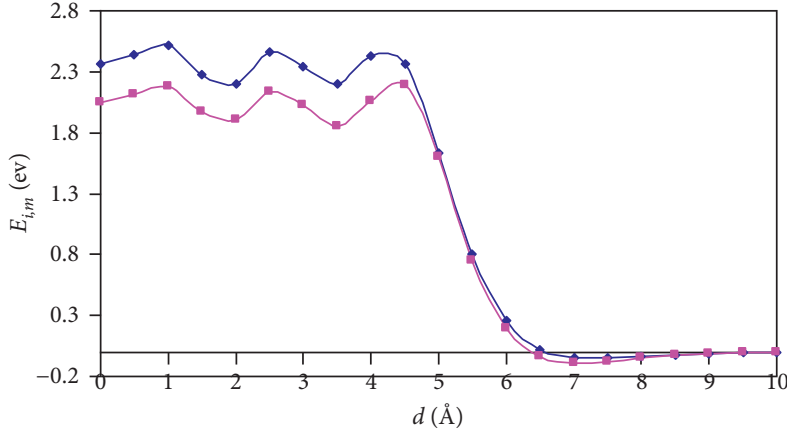

(a)

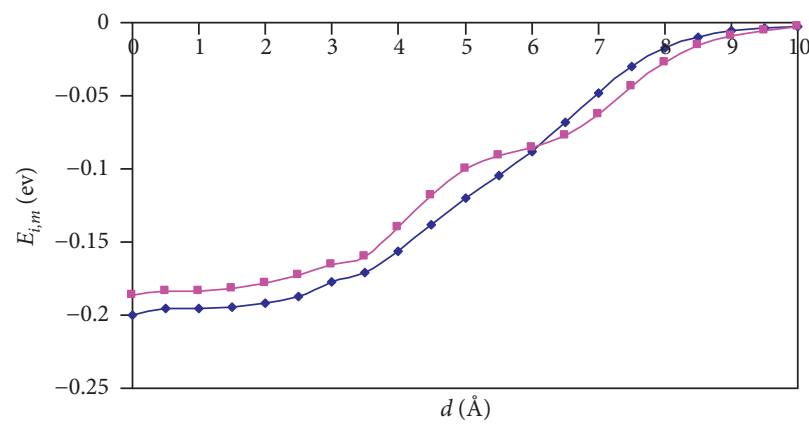

(c)

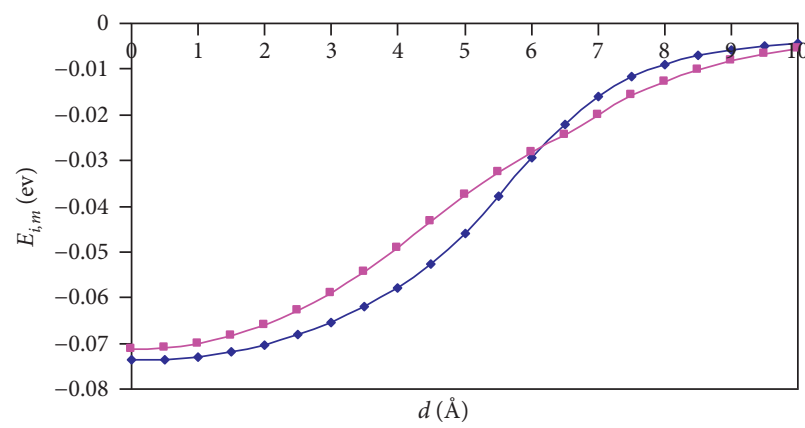

(e)

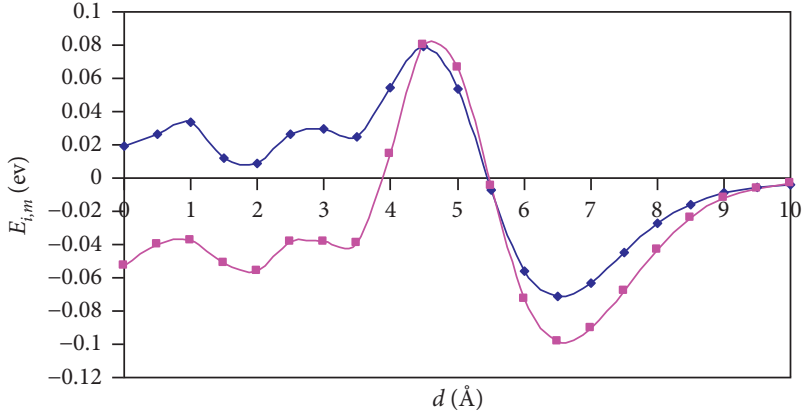

(b)

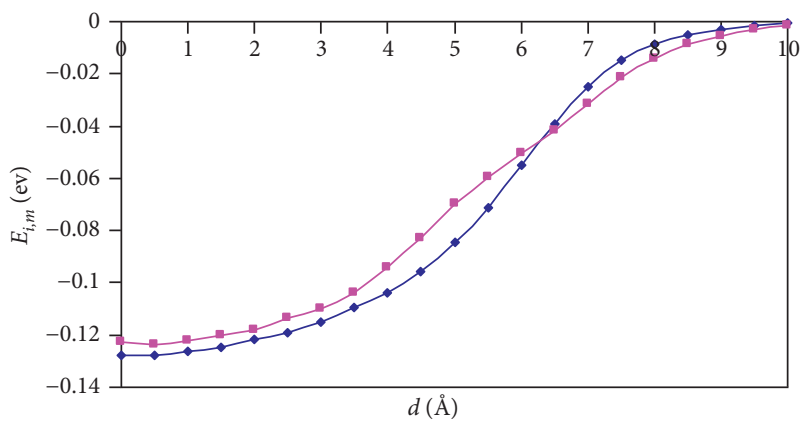

(d)

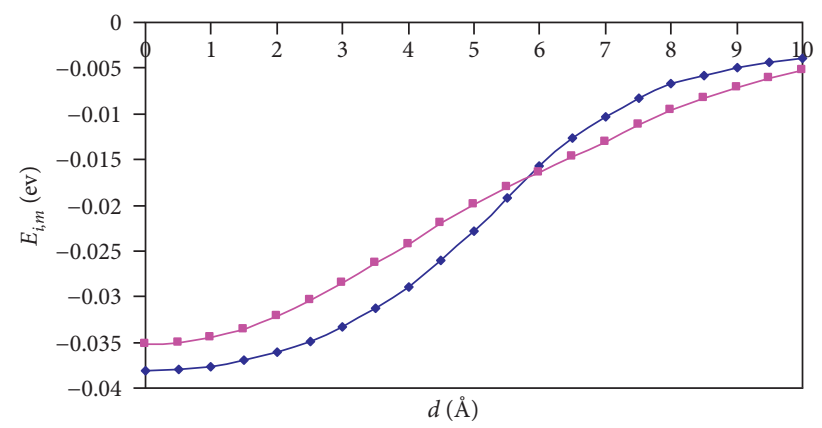

(f)

FIGURE 2: Interaction energies $\left(E_{i, m}, m=1-10\right)$ for the insertion of a hydrogen molecule into $(3,3)-(8,8)$ SWCNTs perpendicularly $(\bullet$, blue) and in parallel $(\mathbf{\square}$, magenta) with the nanotube axis as a function of $d$. (a) $(3,3)$ SWCNT. (b) $(4,4)$ SWCNT. (c) $(5,5)$ SWCNT. (d) $(6,6)$ SWCNT. (e) $(7,7)$ SWCNT. (f) $(8,8)$ SWCNT.

SWCNT which has lower diameter compared with the $(4,4)$ counterpart. For $(3,3)$ and $(4,4)$ SWCNTs, at the distances from 0 to $5 \AA$ (i.e., inside the SWCNTs), one can see the oscillations of $E_{i, m}$ values. As a whole, the orientation of an $\mathrm{H}_{2}$ molecule parallel with the axis of $(3,3)$ and $(4,4)$ SWCNTs is more energetically favorable (Figure 2). For the larger SWCNTs, the entering of $\mathrm{H}_{2}$ is a barrier-free one (Figure 2) for either orientation.

For $(\mathrm{n}, \mathrm{n})$ SWCNTs $(n=5-8)$, the $E_{i, m}$ versus $d$ graphs adopt the "S-shape" character, and we can see that, for the parallel orientation of an $\mathrm{H}_{2}$ molecule, the curve acquires a more slanted shape than for the perpendicular orientation. At a distance of $6 \AA$, there is a point of intersection of the two curves. Thus, one can observe the interesting feature: namely, the hydrogen approaching the nanotubes is more favorable in the parallel orientation, and the introduction is more favorable in the perpendicular orientation.

3.2. Endohedral Complexes of Hydrogen with SWCNTs and Graphene. Table 1 lists predicted binding and interaction energies as well as equilibrium distances between $\mathrm{H}_{2}$ and SWCNTs (or graphene).

There is no sufficient internal space in the smallest considered SWCNTs, $(3,3)$ and $(4,4)$, to effectively adsorb hydrogen molecules. Therefore, $E_{b}$ values are considerably less favorable than those for SWCNTs of larger diameters (Table 1). The $R$ values of 2.00 and $2.75 \AA$ for the parallel 
TABLE 1: PBE-D3/SVP binding $\left(E_{b}\right)$ and interaction energies $\left(E_{i}\right)$ for endohedral complexes of hydrogen and SWCNTs (graphene). The equilibrium distances $(R, \AA)$ are normal between center of mass of a hydrogen molecule and a SWCNT (graphene). All energies are in $\mathrm{eV}$.

\begin{tabular}{lcccccc}
\hline System & \multicolumn{3}{c}{$\begin{array}{c}\text { Parallel } \\
\text { orientation }\end{array}$} & \multicolumn{4}{c}{$\begin{array}{c}\text { Perpendicular } \\
\text { orientation }\end{array}$} \\
& $E_{b}$ & $E_{i}$ & $R^{1}$ & $E_{b}$ & $E_{i}$ & $R$ \\
\hline$(3,3)$ SWCNT & 1.906 & 2.048 & 2.00 & 2.149 & 2.360 & 2.00 \\
$(4,4)$ SWCNT & -0.057 & -0.053 & 2.75 & 0.010 & 0.019 & 2.78 \\
$(5,5)$ SWCNT & -0.195 & -0.187 & 3.05 & -0.199 & -0.200 & 3.28 \\
$(6,6)$ SWCNT & -0.135 & -0.123 & 3.07 & -0.141 & -0.128 & 3.04 \\
$(7,7)$ SWCNT & -0.110 & -0.071 & 3.06 & -0.113 & -0.074 & 3.05 \\
$(8,8)$ SWCNT & -0.077 & -0.035 & 3.54 & -0.076 & -0.038 & 3.62 \\
Graphene & -0.066 & -0.067 & 3.10 & -2 & -0.068 & 3.10 \\
\hline
\end{tabular}

${ }^{1}$ The equilibrium distances $(R)$ are measured between an $\mathrm{H}_{2}$ molecule and the closest wall of a SWCNT; ${ }^{2}$ for graphene, there is only one value of $E_{b}$ as the initial parallel and perpendicular orientations converge to one equilibrium configuration.

orientation of $\mathrm{H}_{2}$ in $(3,3)$ and $(4,4)$ SWCNTs, respectively, are much smaller than those for larger SWCNTs and graphene. The $\mathrm{H}-\mathrm{H}$ bond lengths in the hydrogen molecule disturbed significantly on $\mathrm{H}_{2}$ introduction into $(3,3)$ and $(4,4)$ SWCNTs. Thus, for example, for the $\mathrm{H}_{2}$ molecule which is perpendicularly introduced into $(3,3)$ SWCNT, the changes in the $\mathrm{H}-\mathrm{H}$ bond length are of $0.04 \AA$ ( 5 per cent), whereas for the in-parallel introduced molecule, the variation is of $0.02 \AA$ ( 2.5 per cent). For $(5,5)-(8,8)$ SWCNTs, there are no such large deviations. For $(4,4)$ SWCNT, the parallel orientation of $\mathrm{H}_{2}$ is more energetically favorable than the perpendicular one, although the binding is weak. The reverse situation can be observed for larger SWCNT, where the perpendicular orientations become energy minima. Again, for $(8,8)$ SWCNT, the parallel orientation is more favorable. However, the two orientations are of almost equal energies, and it is possible they both exist.

As a whole, one may observe the increase in $E_{b}$ on going from $(3,3)$ to $(5,5)$ SWCNTs and the following decrease on going from $(5,5)$ to $(8,8)$ SWCNTs, with the maximal $E_{b}$ of -0.195 (parallel orientation) and $-0.199 \mathrm{eV}$ (perpendicular orientation) (Table 1). It can be explained by the very small diameters of the $(3,3)$ and $(4,4)$ SWCNTs which complicate the introduction of hydrogen. $E_{b}$ is unfavorable for the perpendicular orientation of $\mathrm{H}_{2}$ even in the case of $(4,4)$. The larger tubes provide more space for $\mathrm{H}_{2}$ molecules to introduce into and adsorb onto the internal walls of SWCNTs. Besides this, $(5,5)$ SWCNT represents the best diameter/number of neighbor carbon atoms ratio. Indeed, the comprehensive work of Okamoto and Miyamoto [39] showed that the $E_{b}$ values depend significantly on the carbon nanotubes' curvature or, in other words, on the number of neighbor carbon atoms. The ease of the $\mathrm{H}_{2}$ introduction into SWCNTs of large diameters is accompanied by the gradual decline in $E_{b}$. The observed trends in $E_{b}$ changes were also found in the paper of Munusamy and Wheeler [14] on substituted benzenes and in works of Tournus et al. $[15,16]$ on benzene. Finally, the $E_{b}$ values for $(8,8)$ SWCNT, which has the largest diameters and the smallest curvature among studied nanotubes, approach to the values for graphene.

In terms of practical applications, we propose to utilize a number of uniform SWCNTs for hydrogen storage. The methods of the scalable synthesis and separation of SWCNTs can be found elsewhere [40, 41]. Compared with flat graphene-based materials for $\mathrm{H}_{2}$ storage, the $(5,5)$ and $(6,6)$ SWCNTs have the improved $E_{b}$ values. Therefore, these nanotubes require comparatively low pressures to achieve a reasonable storage capacity. The usage of the uniform materials can promote the hydrogen uptake and thus modify the relatively harsh operation mode of existing carbon-based hydrogen storage systems. Our predictions can be used as a guiding reference in employment of high-capacity hydrogen storage materials based on SWCNTs for practical usage.

\section{Conclusions}

We have examined the introduction of hydrogen molecules into SWCNTs, calculated $E_{b}$ and $E_{i}$ values of $\mathrm{H}_{2} / \mathrm{SWCNT}$ complexes, and compared the results with those on graphene. Overall, we reached the following four main conclusions: (i) Hydrogen interacts stronger with the inside of SWCNTs than with graphene. Moreover, for all but the smallest nanotubes, there is a barrier-free energy pathway for formation of these endohedral complexes. (ii) For small $(3,3)$ and $(4,4)$ SWCNTs, $E_{b}$ values are unfavorable owing to very small diameters of these nanotubes; besides this, there are strong interactions between the walls of SWCNTs and hydrogen molecules affecting $\mathrm{H}-\mathrm{H}$ bonds. (iii) SWCNTs of middle diameters $((6,6)$ and especially $(5,5)$ SWCNTs) are the most effective ones in terms of hydrogen storage. (iv) For larger SWCNTs, which have smaller curvatures, the $E_{b}$ values approach to those for graphene.

\section{Conflicts of Interest}

The author declares that there are no conflicts of interest.

\section{Acknowledgments}

This work was partially supported by the base part of the Government Assignment for Scientific Research from the Ministry of Education and Science of Russia (project code: 13.7232.2017/8.9). The author thanks the joint center "Baikal Center of Nanotechnologies" for its facilities.

\section{References}

[1] S. Iijima and T. Ichihashi, "Single-shell carbon nanotubes of 1-nm diameter," Nature, vol. 363, no. 6430, pp. 603-605, 1993.

[2] J. Kong, N. R. Franlin, C. Zhou et al., "Nanotube molecular wires as chemical sensors," Science, vol. 287, no. 5453, pp. 622-625, 2000.

[3] E. S. Snow, F. K. Perkins, E. J. Houser, S. C. Badescu, and T. L. Reinecke, "Chemical detection with a single-walled carbon nanotube capacitor," Science, vol. 307, no. 5717, pp. 1942-1945, 2005. 
[4] C. R. Martin and P. Kohli, "The emerging field of nanotube biotechnology," Nature Reviews Drug Discovery, vol. 2, no. 1, pp. 29-37, 2003.

[5] S. Rahali, Y. Belhocine, J. Touzeau, B. Tangour, F. Maurel, and M. Seydou, "Balance between physical and chemical interactions of second-row diatomic molecules with graphene sheet," Superlattices and Microstructures, vol. 102, pp. 45-55, 2017.

[6] A. Mejri, D. Vardanega, B. Tangour, T. Gharbi, and F. Picaud, "Encapsulation into carbon nanotubes and release of anticancer cisplatin drug molecule," Journal of Physical Chemistry B, vol. 119, no. 2, pp. 604-611, 2015.

[7] A. Gannouni, M. Ouraghi, S. Boughdiri, R. Bessrour, A. Benaboura, and B. Tangour, " $\mathrm{C}_{6} \mathrm{H}_{2} @$ single walled carbon nanotube first principle theoretical study: equivalent temperature confinement effect of carbon nanotubes," Journal of Computational and Theoretical Nanoscience, vol. 9, no. 3, pp. 379-383, 2012.

[8] S. Dargouthi, C. Minot, and B. Tangour, "Structural study of $\mathrm{TiO}_{2}$ nanotube based to the (101) anatase surface," Superlattices and Microstructures, vol. 102, pp. 307-313, 2017.

[9] S. Dargouthi, S. Boughdiri, and B. Tangour, "Stability of $\mathrm{TiO}_{2}$ molecule confined inside a carbon nanotube: a theoretical study," Journal of Computational and Theoretical Nanoscience, vol. 11, no. 5, pp. 1258-1263, 2014.

[10] R. Bessrour, Y. Belmiloud, Z. Hosni, and B. Tangour, "Controlling drug efficiency by encapsulation into carbon nanotubes: a theoretical study of the antitumor cisplatin and the anti-HIV TIBO molecules," AIP Conference Proceedings, vol. 1456, no. 1, pp. 229-239, University of Pavia, Italy, 2-7 September, 2012.

[11] D. A. Britz and A. N. Khlobystov, "Noncovalent interactions of molecules with single walled carbon nanotubes," Chemical Society Reviews, vol. 35, no. 7, pp. 637-659, 2006.

[12] Y.-L. Zhao and J. F. Stoddart, "Noncovalent functionalization of single-walled carbon nanotubes," Accounts of Chemical Research, vol. 42, no. 8, pp. 1161-1171, 2009.

[13] E. S. Zarudnev, S. G. Stepanian, L. Adamowicz, V. S. Leontiev, and V. A. Karachevtsev, "Comparison of noncovalent interactions of zigzag and armchair carbon nanotubes with heterocyclic and aromatic compounds: imidazole and benzene, imidazophenazines, and tetracene," Chemical Physics, vol. 483-484, pp. 68-77, 2017.

[14] E. Munusamy and S. E. Wheeler, "Endohedral and exohedral complexes of substituted benzenes with carbon nanotubes and graphene," Journal of Chemical Physics, vol. 139, no. 9, p. 094703, 2013.

[15] F. Tournus, S. Latil, M. I. Heggie, and J.-C. Charlier, " $\pi$-stacking interaction between carbon nanotubes and organic molecules," Physical Review B, vol. 72, no. 7, p. 075431, 2005.

[16] F. Tournus and J.-C. Charlier, "Ab initio study of benzene adsorption on carbon nanotubes," Physical Review B, vol. 71, no. 16, p. 165421, 2005.

[17] C. Liu, F. Li, M. Lai-Peng, and H.-M. Cheng, "Advanced materials for energy storage," Advanced Materials, vol. 22, no. 8, pp. E28-E62, 2010.

[18] Y. Zhai, Y. Dou, D. Zhao, P. F. Fulvio, R. T. Mayes, and S. Dai, "Carbon materials for chemical capacitive energy storage," Advanced Materials, vol. 23, no. 42, pp. 48284850, 2011.

[19] M. Pumera, "Graphene-based nanomaterials for energy storage," Energy and Environmental Science, vol. 4, no. 3, pp. 668-674, 2011.
[20] B. Szczęśniak, J. Choma, and M. Jaroniec, "Gas adsorption properties of graphene-based materials," Advances in Colloid and Interface Science, vol. 243, pp. 46-59, 2017.

[21] S. Krishnan, R. Vadapoo, K. E. Riley, and J. P. Velev, "Dispersion-corrected density functional theory comparison of hydrogen adsorption on boron-nitride and carbon nanotubes," Physical Review B, vol. 84, no. 16, p. 165408, 2011.

[22] S. Rahali, Y. Belhocine, M. Seydou, F. Maurel, and B. Tangour, "Multiscale study of the structure and hydrogen storage capacity of an aluminum metal-organic framework," International Journal of Hydrogen energy, vol. 42, no. 22, pp. 15271-15282, 2017.

[23] S. Rahali, M. Seydou, Y. Belhocine, F. Maurel, and B. Tangour, "First-principles investigation of hydrogen storage on lead (II)-based metal-organic framework," International Journal of Hydrogen Energy, vol. 41, no. 4, pp. 2711-2719, 2016.

[24] Y. Belmiloud, W. Djitli, H. Abdeldjebar, M. L. Abdelatif, B. Tangour, and M. Brahimi, "First principles calculations of a $\mathrm{H}_{2}$ molecule inside boron-nitrogen nanotubes," Superlattices and Microstructures, vol. 101, pp. 547-558, 2017.

[25] W. F. Gtari and B. Tangour, "A theoretical study of the dihydrogen molecule confined inside carbon nanotubes," International Journal of Quantum Chemistry, vol. 113, no. 21, pp. 2397-2404, 2013.

[26] I. K. Petrushenko, "A DFT study of hydrogen adsorption onto graphene: effect of nitrogen doping," Journal of Nano- and Electronic Physics, vol. 9, no. 3, pp. 03018-1-03018-5, 2017.

[27] I. K. Petrushenko and K. B. Petrushenko, "Physical adsorption of $\mathrm{N}$-containing heterocycles on graphene-like boron nitridecarbon heterostructures: a DFT study," Computational and Theoretical Chemistry, vol. 1117, pp. 162-168, 2017.

[28] D. Cortes-Arriagada, "Adsorption of polycyclic aromatic hydrocarbons onto graphyne: comparisons with graphene," International Journal of Quantum Chemistry, vol. 117, no. 7, p. e25346, 2017.

[29] F. Neese, "The ORCA program system," Wiley Interdisciplinary Reviews: Computational Molecular Science, vol. 2, no. 1, pp. 73-78, 2012.

[30] S. Grimme, "Semiempirical GGA-type density functional constructed with a long-range dispersion correction," Journal of Computational Chemistry, vol. 27, no. 15, pp. 1787-1799, 2006.

[31] S. Grimme, J. Antony, S. Ehrlich, and H. Krieg, "A consistent and accurate $\mathrm{ab}$ initio parametrization of density functional dispersion correction (DFT-D) for the 94 elements $\mathrm{H}-\mathrm{Pu}$," Journal of Chemical Physics, vol. 132, no. 15, p. 154104, 2010.

[32] J. P. Perdew, K. Burke, and M. Ernzerhof, "Generalized gradient approximation made simple," Physical Review Letters, vol. 77, no. 18, pp. 3865-3868, 1996.

[33] H. H. Ansgar Schafer, H. Horn, and R. Ahlrichs, "Fully optimized contracted Gaussian basis sets for atoms Li to Kr," Journal of Chemical Physics, vol. 97, no. 4, pp. 2571-2577, 1992.

[34] N. Faginas-Lago, D. Yeni, F. Huarte, Y. Wang, M. Alcamí, and F. Martin, "Adsorption of hydrogen molecules on carbon nanotubes using quantum chemistry and molecular dynamics," Journal of Physical Chemistry A, vol. 120, no. 32, pp. 6451-6458, 2016.

[35] D. Josa, J. Rodríguez-Otero, E. M. Cabaleiro-Lago, and M. Rellán-Piñeiroa, "Analysis of the performance of DFT-D, M05-2X and M06-2X functionals for studying $\pi \cdots \pi$ interactions," Chemical Physics Letters, vol. 557, pp. 170-175, 2013. 
[36] X. Zhao, Y. Liu, S. Inoue, T. Suzuki, R. O. Jones, and Y. Ando, "Smallest carbon nanotube is $3 \mathrm{~A}$ in diameter," Physical Review Letters, vol. 92, no. 12, pp. 125502-1-125502-3, 2004.

[37] L. Guan, K. Suenaga, and S. Iijima, "Smallest carbon nanotube assigned with atomic resolution accuracy," Nano Letters, vol. 8, no. 2, pp. 459-462, 2008.

[38] M. Pykal, P. Jurecka, F. Karlicky, and M. Otyepka, "Modelling of graphene functionalization," Physical Chemistry Chemical Physics, vol. 18, no. 9, pp. 6351-6372, 2016.

[39] Y. Okamoto and Y. Miyamoto, "Ab initio investigation of physisorption of molecular hydrogen on planar and curved graphenes," Journal of Physical Chemistry B, vol. 105, no. 17, pp. 3470-3474, 2001.

[40] W. G. Reis, R. T. Weitz, M. Kettner et al., "Highly efficient and scalable separation of semiconducting carbon nanotubes via weak field centrifugation," Scientific Reports, vol. 6, Article ID 26259, 2016.

[41] D. Yu, K. Goh, H. Wang et al., "Scalable synthesis of hierarchically structured carbon nanotube-graphene fibres for capacitive energy storage," Nature Nanotechnology, vol. 9, no. 7, pp. 555-562, 2014. 


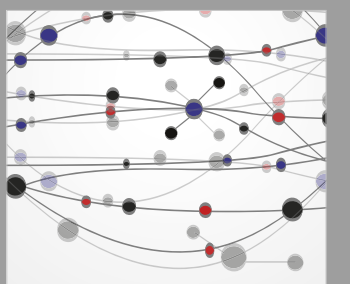

The Scientific World Journal
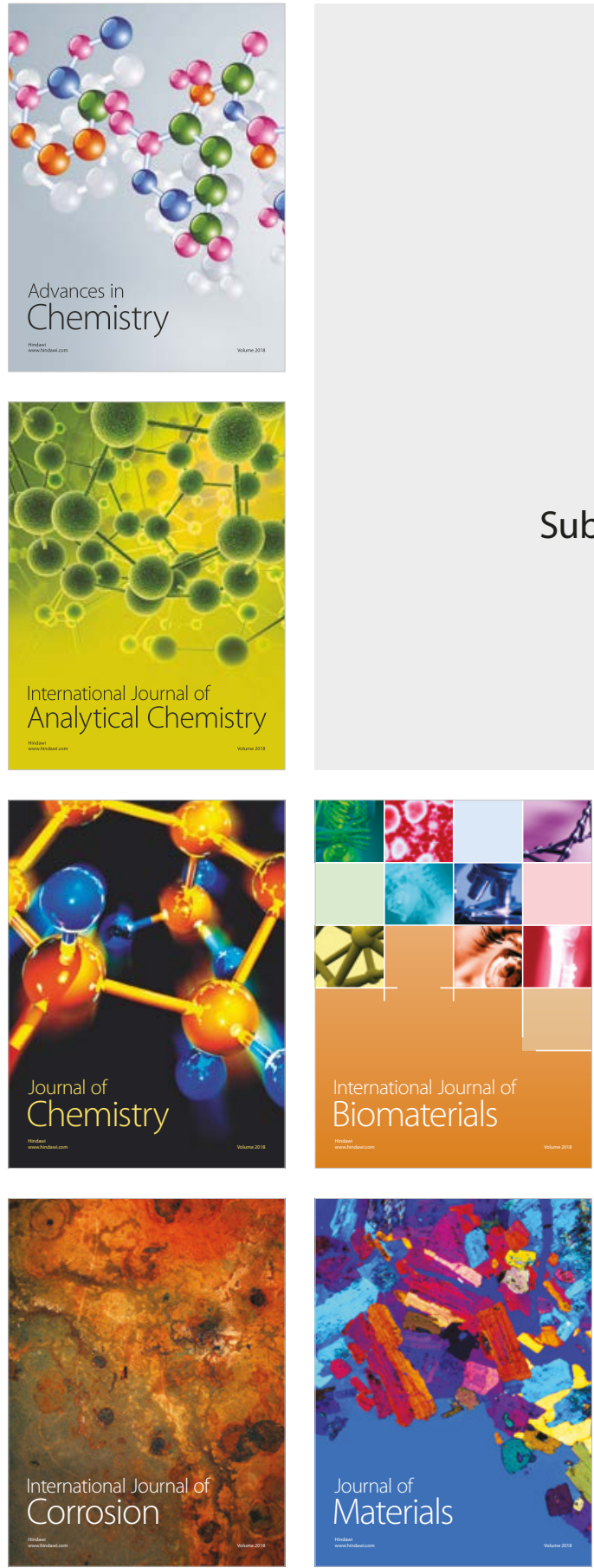

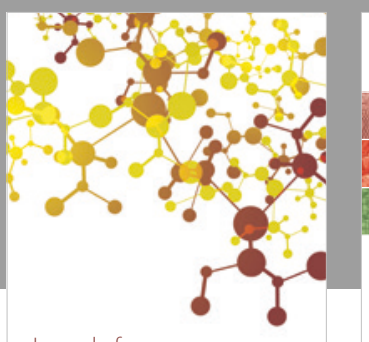

Journal of

Applied Chemistry
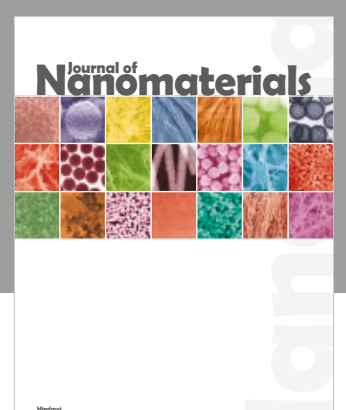

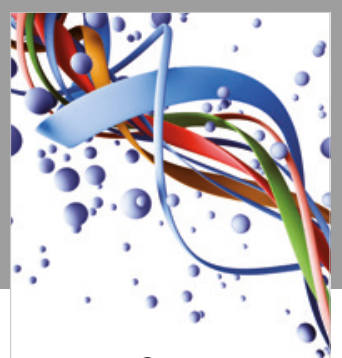

Scientifica

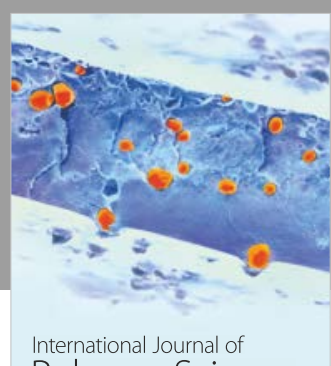

Polymer Science

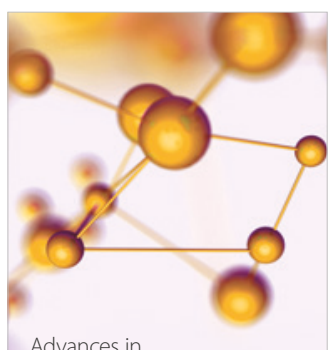

Physical Chemistry
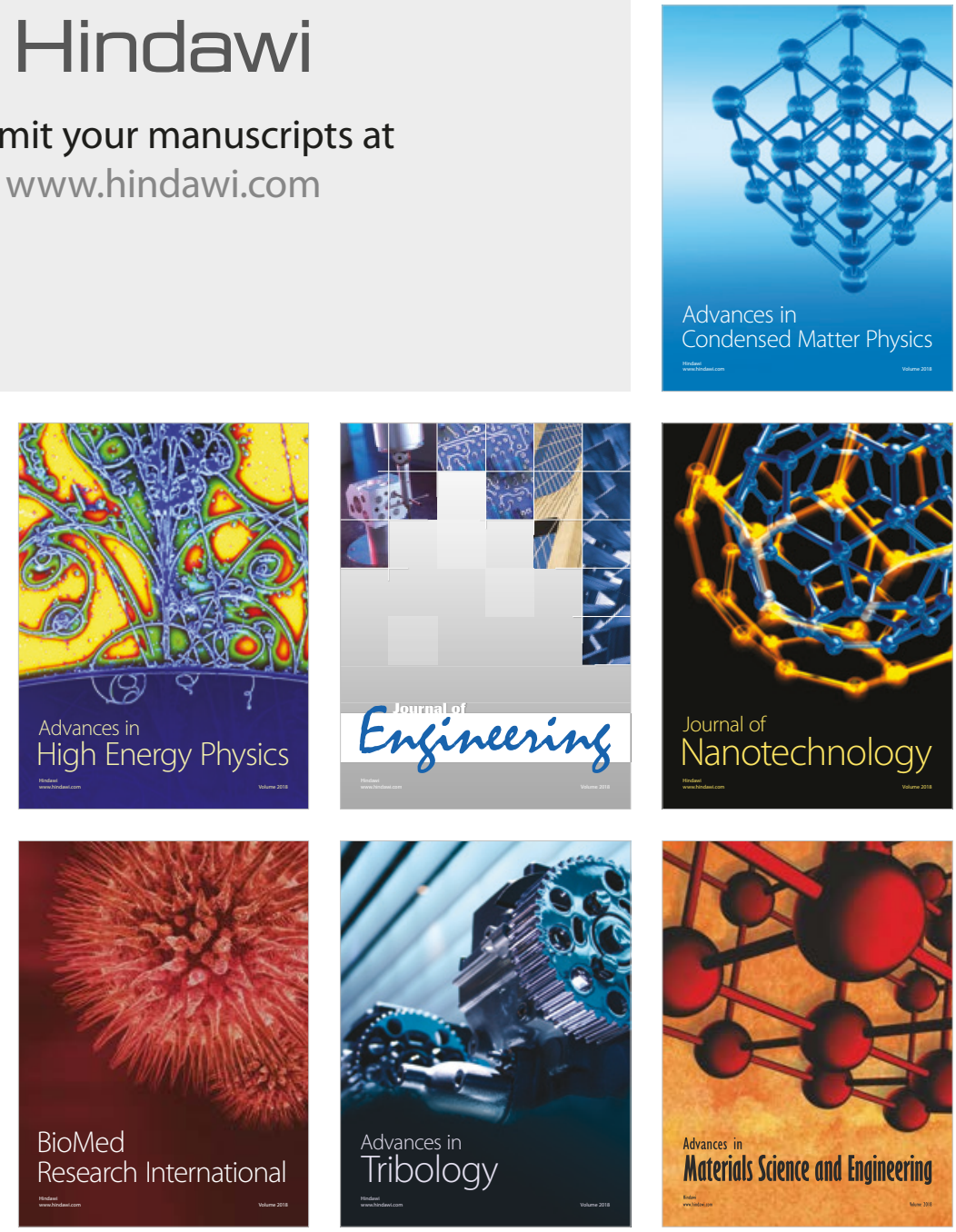